\title{
Pulmonary rheumatoid nodules leading to spontaneous pneumothorax
}

K.R. Dewan ${ }^{1}$, S.K. Nanda ${ }^{2}$, R.S. Twayana ${ }^{3}$

${ }^{1}$ Lecturer, ${ }^{2}$ Associate Professor, ${ }^{3} 1^{\text {st }}$ year Resident, Department of Medicine, College of Medical Sciences, Bharatpur, Nepal.

\begin{abstract}
Several pulmonary diseases are associated with spontaneous pneumothorax, including bronchial asthma, chronic obstructive airway disease, bronchiectasis, pneumonia, abscess, primary or metastatic cancer, and chronic interstitial lung disease. ${ }^{1}$ Spontaneous pneumothorax secondary to rheumatoid nodules which is one of the extra-articular manifestations of rheumatoid arthritis is rare but occasionally described. ${ }^{2}$ There are some reports resulting in spontaneous pneumothorax especially on patients on methotrexate or leflunomide treatment. ${ }^{3}$ We describe a case of rheumatoid arthritis who was on methotrexate therapy from a long time complicating in right sided spontaneous pneumothorax.
\end{abstract}

Key words: Spontanous pneumothorax, rheumatoid arthritis (RA), methotrexate, leflunomide.

\section{Case Report:}

A 45 year old male patient, farmer from Dang district, who was a known case of sero-positive rheumatoid arthritis presented in our hospital with history of breathlessness of 5 days duration which was progressive and mild chest pain of same duration. He was non hypertensive, non diabetic and there was no history of any pulmonary diseases. He was a non smoker and did not consume alcohol and was on methotrexate and steroid therapy since 15 years. On clinical examination, patient was dyspnoic and lying on right lateral position. $\mathrm{BP}$ was $110 / 70 \mathrm{~mm} \mathrm{Hg}$ on right arm with pulse rate of $110 / \mathrm{min}$, regular with normal volume and character. Patient was afebrile with respiratory rate of 48/min regular and normal JVP. Chest examination revealed less expansion of right side Correspondence: K.R. Dewan E-mail: dewansantosh@yahoo.com of the chest, hyper-resonant on percussion and absent breath sound on auscultation on the same side. Other systems were normal. There were signs of steroid toxicity like moon face, buffalo hump, wasting of thigh muscles, purple straie over abdomen, bruises on upper and lower extremities and joint deformities typical of rheumatoid arthritis.

Blood hemogram showed WBC-12.6 x $10^{6}$ cumm with N-78\%, L-19\%, E-03\%. ESR was 73 mm/hr. Blood sugar, renal, liver and thyroid function test were all within normal range. Serum $\mathrm{RA}$ factor and $\mathrm{C}$ reactive protein was positive. ECG showed sinus tachycardia. Chest xray revealed right sided massive pneumothorax with mediastinal shift towards left side and pulmonary nodule on left upper zone. CT scan was planned but was not done due to financial problem of the patient. 
Chest tube was inserted and was removed after 10 day. Patient was discharged with modification of treatment.

a

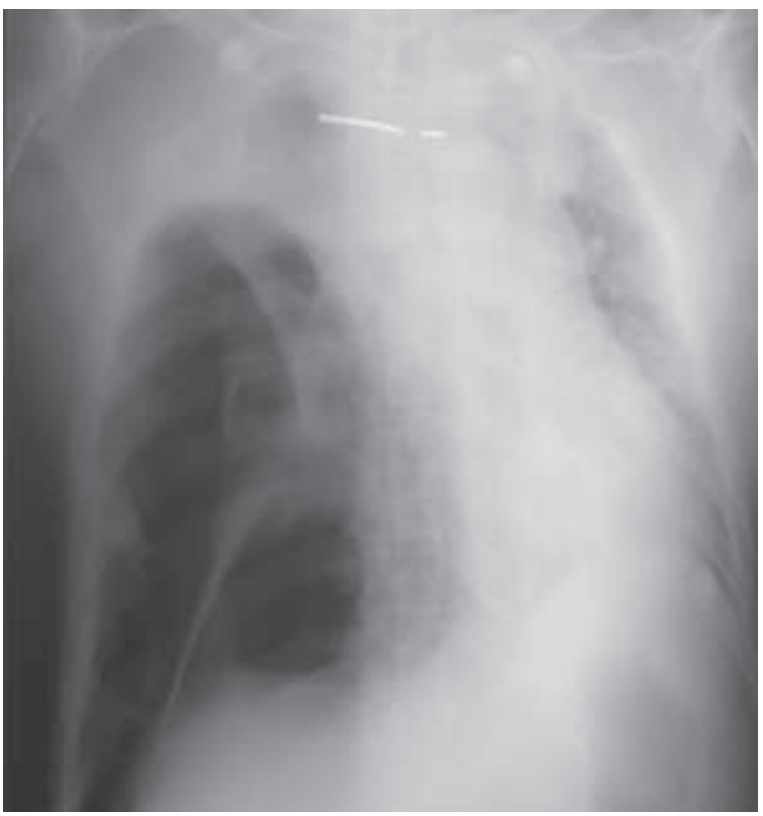

Figure 1: (a) Showing localized pneumothorax in right lung field with mediasternal shift to the

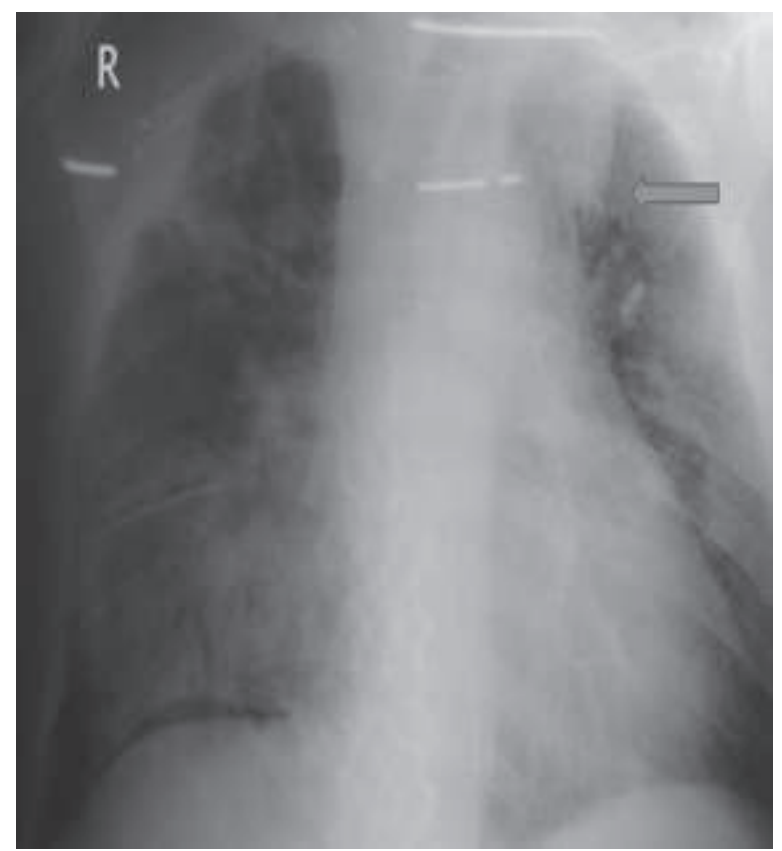

Figure 1: (b) Showing rheumatoid nodule in left upper zone.

\section{Discussion}

Rheumatoid arthritis (RA) is an autoimmune chronic inflammatory disease that affects the synovial membrane, leading to bone damage and joint destruction. Pulmonary manifestations varying from pleural effusions, pleural thickening, mottling, reticulation, and chronic fibrosis have been observed in patients with rheumatoid arthritis. One of the most common extra-articular pulmonary manifestations of RA is the rheumatoid nodule, which is found in approximately less than $0.5 \%$ of patients with this disease. ${ }^{4}$ Rheumatoid nodules in the lung are most common in patients who have a positive test for rheumatoid factor and also have subcutaneous rheumatoid nodules. In 1953, Caplan first described a syndrome of nodular lung fibrosis which was associated with rheumatoid arthritis in coal-miners and the prevalence of rheumatoid arthritis was increased amongst men with complicated pneumoconiosis. ${ }^{5} \mathrm{He}$ also discovered the characteristic multiple bilateral nodules on chest radiographs of coal miners with RA. ${ }^{6}$ The rheumatoid nodule is the most distinctive but least common lesion in the pulmonary parenchyma in patients with rheumatoid lung disease. ${ }^{7}$ These nodules may develop in the pleura and pericardium and occasionally form an exophytic mass that may obstruct a bronchus and mimics a bronchogenic carcinoma at bronchoscopy ${ }^{8}$ These nodules usually occur at the periphery of the lung, just beneath the pleura, and sometimes can cause bronchopleural fistula, abscess formation, cavitation and pneumothorax. ${ }^{9}$

In our case, most probably necrobiotic process occurred in the nodules on the pleura leading to spontaneous pneumothorax. The event was insidious 
K.R. Dewan et al, Pulmonary rheumatoid nodules leading to spontaneous pneumothorax

on onset and almost painless which was similar in other case reports ${ }^{10}$ also suggesting the gradual erosion of visceral pleura overlying the nodule rather than the usually sudden and painful acute pneumothorax in other condition like emphysema.

Some authors have reported similar cases associated with eosinophilia indicating a more aggressive course of RA with extra-articular complications. ${ }^{8,11}$ But in our case the eosinophil count was within normal range (03\%). One possibility of having normal eosinophil count may be due to prolonged use of steroid as our patient had signs of steroid toxicity.

There are some anecdotal reports suggesting that methotrexate and leflunomide exacerbating subcutaneous nodulosis in RA but the mechanism is still unknown. ${ }^{12,13}$ Methotrexate is a first line disease modifying drug in rheumatoid arthritis but its role in extra-articular complications is less clear and moreover it carries a significant risk of pulmonary toxicity. ${ }^{14}$ Leflunomide is a new generation immunosuppressive drug and its side effects like nausea, vomiting, diarrhea, upper respiratory tract infection, hypertension and hepatotoxicity have been widely known. One case was reported in patient with RA who developed a pulmonary abscess due to leflunomide. ${ }^{15}$ Our patient was under methotrexate therapy from a long time. So we assumed that the other factor which leaded to this pulmonary complication might be methotrexate aggravating the pulmonary disease causing enlargement of the pulmonary cavitation leading to spontaneous pneumothorax. In view of these reports, we gave disease-modifying treatment with hydroxychloroquine instead of methotrexate to our patient because of concern that this might aggravate pulmonary nodulosis.

\section{Conclusion}

Pulmonary nodules are a rare manifestation of RA and sometimes may cause spontaneous pneumothorax depending on their location. The role of methotrexate in exacerbating the pulmonary nodules in RA and causing spontaneous pneumothorax is still not clear.

\section{References}

1. R.G. Fraser J.A. Pare Diagnosis of Diseases of The Chest Philadelphia: Saunder. 1970;867-9:1159-61.

2. W. Hindle, D.A.H.Yates. Pyopneumothorax complicating rheumatoid lung disease. Ann Rheum Dis 1965;24:57-60.

3. M. Abu-Abeeleh, N. Shourbasi, M. Nofal et al. Complicated Spontaneous pneumothorax in a patient with rheumatoid arthritis. European Journal of Scientific Research. 2009;36:130-3.

4. H. Adelman, E. Dupont, M. Flannery et al. Case report: recurrent pneumothorax in a patient with rheumatoid arthritis. Am J Med Sci. 1994;308:171-2.(ㅁ)

5. A. Arnoff, G.L. Bywaters, G.R. Fearnly. Lung lesion in rheumatoid rthritis. BMJ.1955;228-32.

6. S.I. Byrd, B.A. Case, D.W. Boulware. Pulmonary Manfestations of Rheumatic Disease. Postgrad Med 1993;93:149-51,154-6,159-61.

7. C. Fellbaum, W. Domej, H. Popper. Rheumatoid arthritis with extensive lung lesions. Thorax 1989;44:70-1.

8. T.S. Johnson, P. White, S.T. Weiss et al. Endobronchial necrobiotic nodule antedating rheumatoid arthritis. Chest 1982;82:199- 200. 
Journal of College of Medical Sciences-Nepal,2010,Vol-6,No-4

9. U.B.Parakash. Respiratory Manifestation Of Systemic Disease .Rheumatologic And Vascular Disease. Post GradMed 1984;76:119-25.

10. A.J. Crisp, R.D. Armstrong, R. Grahame et al. Rheumatoid lung disease, pneumothorax, and eosinophilia. Annals of the Rheumatic Diseases 1982;41:137-40

11. R.J. Winchester, S.D. Litwin, D. Koffler et al. Observations on the eosinophiia of certain patients with rheumatoid arthritis. Arthritis Rheum 1971;14: 650-65.

12. I. Gotsman, A. Goral, S. Nusair. Secondary spontaneous pneumothorax in a patient with pulmonary nodules during treatment with methotrexate. Rheumotology 2001; 40:350-1

13. H. Adelman, E. Dupont, M. Flannery et al. Case report: recurrent pneumothorax in a patient with rheumatoid arthritis. Am J. Med. Sci. 1994; 308:171-2.

14. B. Combe, C. Didry, M. Gutierrez et al. Accelerated nodulosis and systemic manifestations during methotrexate therapy for rheumatoid arthritis. Eur $J$ Med. 1993;2:153-6.

15. H. Ulusoy, A. Bilgici, O. Kuru et al. Pulmonary abscess due to leflunomide use in rheumatoid arthritis: a case report. Rheumatol Int. 2005;25:139-42. 